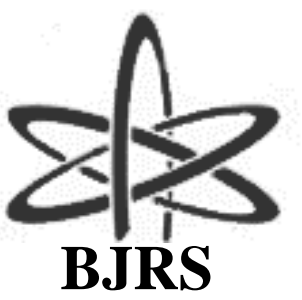

\author{
BRAZILIAN JOURNAL \\ $\mathrm{OF}$ \\ RADIATION SCIENCES \\ 06-02-B (2018) 01-13
}

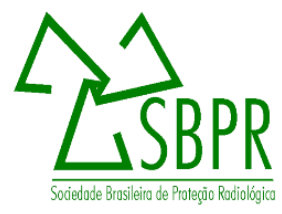

\title{
Avaliação das doses no cristalino de indivíduos ocupacionalmente expostos em um serviço de PET/CT
}

\author{
Fischer A. C. F. S., Moreira M. N., Bacelar A. \\ Hospital de Clínicas de Porto Alegre - HCPA \\ R. Ramiro Barcelos n. 2350, Santa Cecilia, CEP 90035-903, Porto Alegre-RS/Brasil \\ acfischer@hcpa.edu.br
}

\begin{abstract}
RESUMO
O objetivo do estudo é avaliar a dose recebida no cristalino pelo Indivíduo Ocupacionalmente Exposto (IOE) na área de PET/CT do Serviço de Medicina Nuclear (SMN) de um Hospital Escola público de porte especial, determinando a necessidade, ou não, da implementação de um programa rotineiro de monitoração e do uso de dosímetro específico para esse fim. Os IOEs (n=07) foram acompanhados durante sua rotina de trabalho por um mês e monitorados com dosímetros OSL, calibrados em Dose Individual $\left(\mathrm{H}_{\mathrm{x}}\right)$, posicionados na região dos olhos e do tórax, para estimativa das doses equivalente de cristalino $\left(\mathrm{H}_{\text {lens }}\right)$ e efetiva de corpo inteiro (E), respectivamente. Estimou-se a potencial $H_{\text {lens }}$ anual a partir da extrapolação dos valores de $H_{\text {lens }}$ : é improvável que o limite de $20 \mathrm{mSv} / a n o$ seja atingido, entretanto 04 IOEs ultrapassaram o nível de investigação anual. As razões $H_{\text {lens }} / E(0,26-0,91)$ indicam que deveria ser adotado o uso de um dosímetro específico. Entretanto, levando-se em consideração o fator econômico relacionado ao conceito de otimização da radioproteção, a adoção de um dosímetro adicional significaria um aumento de custo injustificável. Logo, conclui-se que, nas condições de trabalho avaliadas no SMN deste hospital, é necessária a implementação de um programa de monitoração, no qual a $\mathrm{H}_{\text {lens }}$ seria estimada a partir dos valores de $\mathrm{E}$ individuais.
\end{abstract}

Palavras-chave: Dosimetria, Cristalino, PET/CT, Proteção Radiológica.

ISSN: 2319-0612

Accepted 2018-07-10 


\begin{abstract}
The aim of this study was to evaluate the Eye Lens Dose received by the Occupationally Exposed Individual (OEI) in the PET/CT area of the Nuclear Medicine Service (NMS) of a Public Hospital, determining the need (or not) of implementation of a routine monitoring program and the use of a specific dosimeter for this purpose. These OEIs $(n=07)$ were followed during their work routine for one month and monitored with OSL dosimeters, calibrated in Individual Dose $\left(\mathrm{H}_{\mathrm{x}}\right)$, positioned in the eye and chest regions, to estimate the Eye Lens $\left(H_{\text {lens }}\right.$ ) and whole-body (E) Doses, respectively. The annual $H_{1}$ dose was estimated from the extrapolation of the $\mathrm{H}_{\text {leans }}$ values: it is unlikely that the limit of $20 \mathrm{mSv} /$ year is reached however 04 OEIs have exceeded the Brazilian annual investigation level. The $\mathrm{H}_{\text {lens }} / \mathrm{E}$ ratios (0.26-0.91) indicate that the use of a specific dosimeter should be adopted. However, taking into account the economic factor related to the concept of radioprotection optimization, the adoption of an additional dosimeter would mean an unjustifiable cost increase. Therefore, under the working conditions assessed in this NMS, it is necessary to implement a monitoring program in which $H_{\text {lens }}$ doses could be estimated from the individual $E$ dose values.
\end{abstract}

Keywords: Dosimetry, Lens, PET/CT, Radiation Protection.

\title{
1. INTRODUÇÃO
}

No Brasil, o limite anual de dose equivalente no cristalino foi reduzido de $150 \mathrm{mSv}$ para $20 \mathrm{mSv}$ em 24 de agosto de 2011 [1], atendendo ao Comunicado "Statement on Tissue Reactions" da Comissão Internacional de Proteção Radiológica (ICRP, do inglês International Commission on Radiological Protection) [2]. Através desse Comunicado a ICRP altera o limiar de dose absorvida pelo cristalino do olho para 0,5 Gy em decorrência de evidências epidemiológicas, então recentes, que sugeriram que o limiar de dose de efeitos com manifestação tardia (usualmente denominados de efeitos determinísticos), incluindo a catarata, seriam menores do que os previamente considerados; e, por conseguinte, reduz o limite de dose equivalente no cristalino para $20 \mathrm{mSv} / a n o$ (média no período de cinco anos, desde que nenhum ano ultrapasse $50 \mathrm{mSv}$ ).

Dessa redução de limite emerge a necessidade de avaliação da dose equivalente recebida no cristalino dos indivíduos ocupacionalmente expostos (IOEs) das áreas de radiologia intervencionista e medicina nuclear, uma vez que tais trabalhadores têm possibilidade de exceder, em condições normais de trabalho, o limite anual revisado [3]. Entretanto, mesmo com a orientação da Comissão 
Nacional de Energia Nuclear (CNEN) de que os procedimentos adotados para estimativa da dose no cristalino sejam descritos no Plano de Proteção Radiológica (PPR) [4], ainda não há um método de dosimetria do cristalino certificado no Brasil [5].

O Serviço de Medicina Nuclear (SMN) avaliado pertence a um Hospital Escola público de porte especial. Iniciou suas atividades em 1972, realizando exames de cintilografia através da administração de radiofármacos, marcados principalmente com Tc-99m - emissor puramente gama com energia de 140 keV. Em 2015, entrou em operação o equipamento de PET/CT (sistema híbrido de Tomografia por Emissão de Pósitrons - PET - e Tomografia Computadorizada por Raios X CT), técnica diagnóstica que emprega radionuclídeos emissores de pósitrons, principalmente o Flúor-18 (F-18, emissor de partículas beta positivas de 634 keV que geram fótons de aniquilação de $511 \mathrm{keV})$. O emprego desses radionuclídeos com maior energia aumentou a probabilidade de acúmulo de doses significativas nas extremidades e cristalino dos IOEs envolvidos na manipulação e administração do material radioativo ao paciente. Uma medida de radioproteção adotada pelo SMN foi a delimitação da área física e fluxo de trabalho/pacientes dos exames cintilográficos tradicionais e dos estudos de PET/CT. Assim, o SMN foi setorizado em três áreas fisicamente delimitadas: Área Comum, Medicina Nuclear e PET/CT. Entretanto, nada foi definido em relação à avaliação da dose do cristalino.

É consenso entre os pesquisadores que o limite anual de dose equivalente no cristalino não é excedido em decorrência unicamente da manipulação do F-18 [6-8]. Contudo, nem todos os autores concordam com a necessidade do emprego de um monitor específico para a avaliação da dose [6-10]. Cabe ao Supervisor de Proteção Radiológica (SPR) estabelecer o método de estimativa que será adotado.

Esse trabalho pretende avaliar a dose recebida no cristalino pelo IOE durante sua rotina de trabalho na área de PET/CT do SMN em questão, determinando a necessidade, ou não, da implementação de um programa rotineiro de monitoração da dose no cristalino; e do uso de um dosímetro específico para esse fim. 


\section{MATERIAIS E MÉTODOS}

Este estudo é produto do Trabalho de Conclusão da Residência (TCR) em Área Profissional da Saúde (Física Médica, ênfase em Medicina Nuclear) do Hospital avaliado e foi aprovado pelo Comitê de Ética e Pesquisa do Hospital pelo parecer nº 2.198.658.

Todos os IOEs do SMN que prestam atendimento direto a pacientes que realizam o exame de PET/CT $(n=07)$ foram convidados a participar da pesquisa e aceitaram voluntariamente. Seus consentimentos foram obtidos por escrito através da assinatura do Termo de Consentimento Livre e Esclarecido (TCLE).

As equipes de trabalho do SMN, monitoradas neste estudo, são constituídas e responsáveis, principalmente, por: Equipe Farmacêutica $(\mathrm{EF})$, fracionamento do radiofármaco - 02 farmacêuticos; Equipe de Enfermagem (EE), assistência ao paciente e administração do radiofármaco - 01 enfermeira e 02 técnicas em enfermagem; e Equipe técnica em Radiologia (ER), posicionamento do paciente na maca de exame e aquisição do estudo de PET/CT - 02 tecnólogas em Radiologia.

Cada IOE utilizou, durante o desempenho de suas tarefas exclusivamente na área do PET/CT, dois dosímetros extras: um dosímetro acoplado entre as lentes dos óculos de proteção de acrílico (Figura 1), disposto na região da glabela; e um dosímetro de corpo inteiro adicional, posicionado na altura do tórax, além dos dosímetros habitualmente usados no SMN (tórax e pulseira).

Figura 1: Dosímetro de cristalino acoplado aos óculos de proteção individual (a) e dosímetro

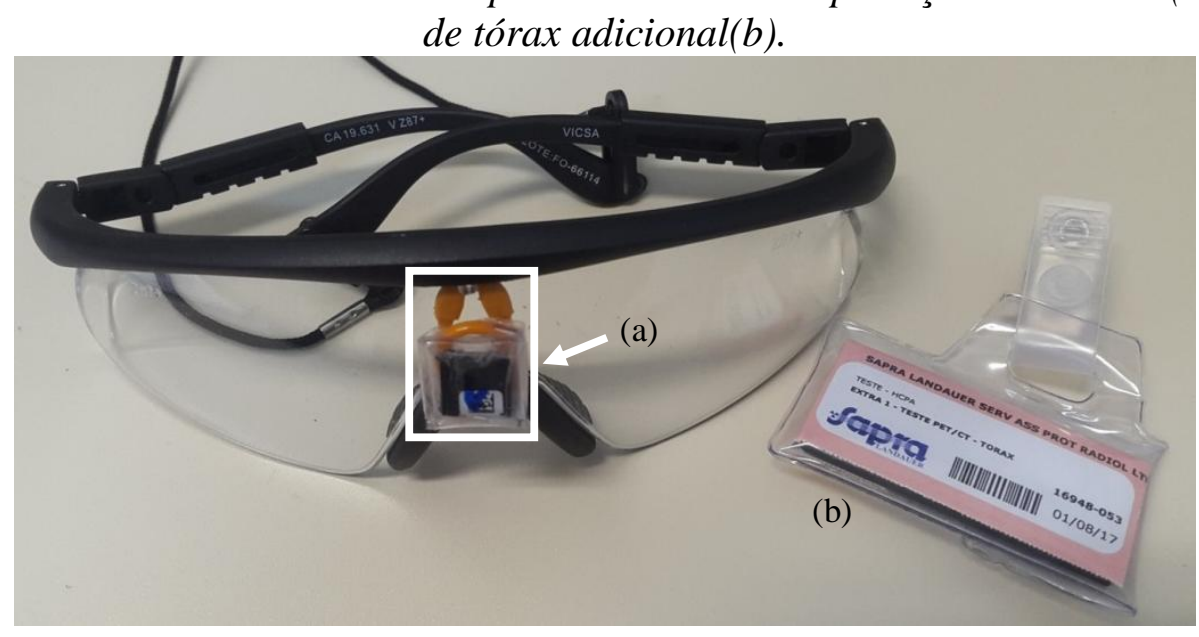


Os dosímetros extras foram fornecidos pela empresa SAPRA LANDAUER - Serviços de Assessoria e Proteção Radiológica. Tais monitores possuíam detectores com tecnologia de luminescência opticamente estimulada (Landauer, França), calibrados em Dose Individual $\left(\mathrm{H}_{\mathrm{x}}\right)$, grandeza operacional adotada no Brasil para monitoração individual externa para feixe de fótons no período em que a coleta de dados foi realizada [11] - agosto a setembro de 2017.

Os laboratórios de dosimetria brasileiros eram certificados pelo CASEC/IRD/CNEN ${ }^{1}$ somente para prestação de serviço de monitoração individual externa de corpo inteiro para feixe de fótons [5]. Não havia, no Brasil, monitores individuais comercialmente disponívéis que fossem testados e calibrados em termos de $\mathrm{H}_{\mathrm{p}}(3)$, grandeza operacional utilizada para estimativa de dose equivalente no cristalino $\left(\mathrm{H}_{\text {lens }}{ }^{2}\right)$ igual ao equivalente de dose individual a uma profundidade de $3 \mathrm{~mm}$ da superfície $[12, .13]$. Apesar de $\mathrm{H}_{\mathrm{p}}(3)$ ser o método mais acurado para monitoração de $\mathrm{H}_{\text {lens }}$, a Agência Internacional de Energia Atômica (IAEA, do inglês International Atomic Energy Agency) cita, no TECDOC n. 1731: Implications for Occupational Radiation Protection of the New Dose Limit for the Lens of the Eye [13], que dosímetros calibrados em outras grandezas operacionais podem ser empregados para estimar um valor conservador para $\mathrm{H}_{\mathrm{p}}(3)$, caso o uso de $\mathrm{H}_{\mathrm{p}}(3)$ seja inviável, contanto que informações sobre o campo de radiação do local de trabalho sejam conhecidas.

O monitor empregado na avaliação da dose no cristalino nesse trabalho (NanoDot ${ }^{\mathrm{TM}}$ ) é constituído de um cristal de $4 \mathrm{~mm}$ de diâmetro de óxido de alumínio dopado com carbono $\left(\mathrm{Al}_{2} \mathrm{O}_{3}: \mathrm{C}\right)$, coberto por uma proteção de plástico de $1,0 \times 1,0 \times 0,2 \mathrm{~cm}$. O monitor de corpo inteiro $\left(\right.$ InLight $\left.^{\circledR}\right)$, usualmente comercializado, é composto por 04 desses cristais, acomodados em um invólucro de plástico de 5,0 x 2,3 x 0,6 cm que contém filtros (janela aberta, alumínio, cobre e plástico) que permitem a identificação da energia e tipo de radiação incidente.

\footnotetext{
${ }^{1}$ Lista de Laboratórios certificados para prestação de serviço de monitoração individual externa de corpo inteiro para feixe de fótons pelo CASEC/IRD/CNEN - Comitê de Avaliação de Serviço de Ensaio e Calibração do Instituto de Radioproteção e Dosimetria/CNEN. Situação em novembro de 2017.

${ }^{2} \mathrm{H}_{\text {lens }}$ é a sigla utilizada internacionalmente para Dose Equivalente no Cristalino.
} 
A coleta de dados foi realizada por um (01) mês e, neste período, acompanhou-se a rotina de trabalho dos IOEs, tabelando as atividades manipuladas de material radioativo por cada IOE. Posteriormente, os dosímetros foram recolhidos e enviados para leitura das doses na empresa fornecedora.

Para análise, os valores individuais de dose efetiva de corpo inteiro (E) e $\mathrm{H}_{\text {lens }}$ recebidos foram normalizados pela atividade total de F-18 manipulada por cada IOE.

Visando a definição da necessidade de implementação de um programa rotineiro de monitoração de dose do cristalino foi estimada a dose anual recebida no cristalino por cada IOE, a partir da extrapolação dos valores de dose coletados (considerando uma carga de trabalho mensal de 100 exames de PET/CT).

Por fim, foram determinados os valores médios $\mathrm{E}$ e $\mathrm{H}_{\text {lens }}$, por equipe, para obtenção da razão entre os resultados. A partir dessa comparação avaliou-se a necessidade da utilização de um dosímetro específico para a estimativa da dose do cristalino, de acordo com o proposto por Kopec e colaboradores [9], Kubo e colaborador [10] e Dabin [6].

\section{RESULTADOS E DISCUSSÕES}

No período de um mês foram realizados 63 pacientes de PET/CT oncológico (15 dias de atendimento, em média 4,2 exames/dia). A atividade média de [F-18]FDG (Fludesoxiglicose) injetada por paciente $\left(\mathrm{A}_{\text {méd }}\right)$ foi $315,36 \mathrm{MBq}(8,52 \mathrm{mCi})$. Entre pacientes adultos $(\mathrm{n}=58)$ a $\mathrm{A}_{\text {méd }}$ foi de $325,93 \mathrm{MBq}(8,81 \mathrm{mCi})$ e entre os pediátricos $(\mathrm{n}=5), 192,70 \mathrm{MBq}(5,21 \mathrm{mCi})$. Os dados registrados, por equipe de trabalho, encontram-se na Tabela 1.

Os valores de $\mathrm{E}$ e $\mathrm{H}_{\text {lens }}$, normalizados pela atividade manipulada por cada IOE monitorado, encontram-se graficados na Figura 2. Percebe-se que a equipe que recebeu maior $\mathrm{H}_{\mathrm{lens}}$ foi a EE, responsável pela administração do material radioativo aos pacientes; e que, como esperado, os IOEs que receberam menor $\mathrm{H}_{\text {lens }}$ pertencem à ER: a ER somente mantém contato com o paciente injetado após o período de captação de aproximadamente 60 minutos, durante o posicionamento/retirada do paciente na mesa de exames. 
Tabela 1: Relação do número de pacientes de PET/CT oncológicos atendidos e o total de atividade manipulada por equipe no período observado.

\begin{tabular}{cccc}
\hline Equipes & IOEs & $\mathbf{N}^{\mathbf{0}}$ de Pacientes $^{\mathbf{a}}$ & A $_{\text {Manip. }}{ }^{\mathbf{b}}[\mathbf{G B q}(\mathbf{m C i})]$ \\
\hline \multirow{2}{*}{$\mathbf{E F}^{\mathbf{c}}$} & EF1 & 18 & $5,62(151,88)$ \\
& EF2 & 44 & $13,85(374,38)$ \\
Média & - & $\mathbf{9 , 7 4}(\mathbf{2 6 3 , 1 3})$ \\
\hline \multirow{2}{*}{ EE } & EE1 & 20 & $6,30(170,22)$ \\
& EE2 & 11 & $3,64(98,51)$ \\
& EE3 & 20 & $6,23(168,30)$ \\
Média & - & $\mathbf{5 , 3 9}(\mathbf{1 4 5 , 6 8})$ \\
\hline & ER1 & 41 & $13,59(367,39)$ \\
& ER2 & 22 & $6,27(169,58)$ \\
& Média & - & $\mathbf{9 , 9 3}(\mathbf{2 6 8 , 4 8})$ \\
\hline
\end{tabular}

${ }^{a}$ Número de exames de PET/CT em que os dosímetros adicionais da pesquisa foram utilizados pelos IOEs de cada equipe de trabalho.

${ }^{\mathrm{b}} \mathrm{A}_{\text {Manip }}=$ Atividade manipulada. Considerada como a atividade de [F-18]FDG fracionada/administrada pela equipe técnica, desprezando o decaimento radioativo.

${ }^{\mathrm{c}}$ Desconsiderou-se a atividade contida no frasco de material radioativo no momento do fracionamento do material a ser injetado.

Figura 2: Valores de E e de $H_{\text {lens }}$ recebidos por cada IOE monitorado (identificado por numeração sequencial após a sigla da equipe a que pertence).

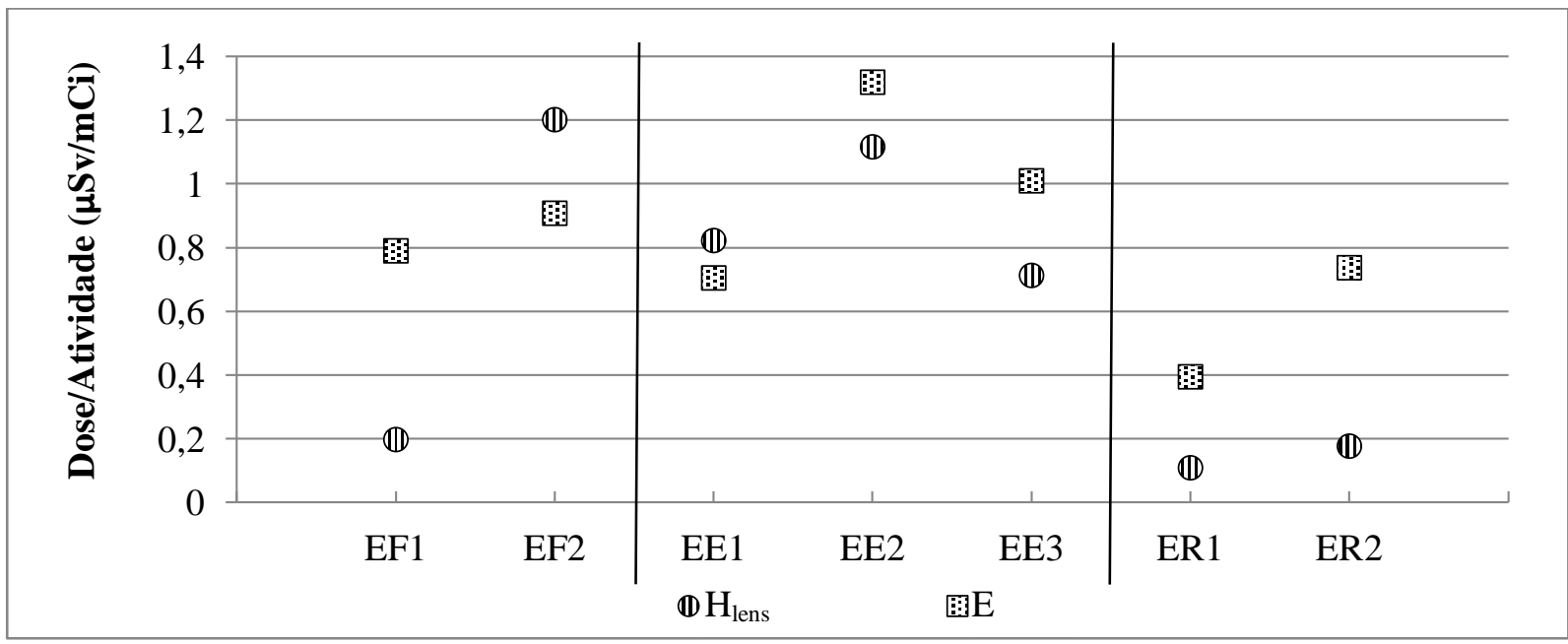


Apenas dois IOEs receberam $\mathrm{H}_{\text {lens }}$ mais elevadas que sua respectiva E: EF2 e EE1. Durante o acompanhamento das rotinas observou-se que o EF2, ao fracionar a atividade de [F-18]FDG a ser injetada no paciente, aproximava a seringa (fonte radioativa) do visor plumbífero para realização de ajustes como retirada de ar e troca de agulhas, causando uma maior exposição do cristalino. Isso pode ser explicado pelo curto tempo de experiência (07 meses ininterruptos) em PET/CT do IOE que, antes de uma colega iniciar licença gestação, apenas cobria folgas ou férias dos demais farmacêuticos do SMN. Optou-se por não realizar nenhuma intervenção, pois o objetivo, nessa etapa do trabalho, era avaliar a exposição sofrida por cada IOE durante os procedimentos realizados em sua rotina de trabalho. Observou-se, também, que o EE1 não utilizava os óculos de acrílico com o dosímetro de cristalino na região dos olhos durante assistência ao paciente (acompanhamento do paciente ao banheiro/sala de exames), deixando-o pendurado no pescoço, próximo ao dosímetro de corpo inteiro. O IOE, mesmo quando solicitado, não posicionava o óculos corretamente, mostrandose não colaborativo. Logo, o valor de $\mathrm{H}_{\text {lens }}$ obtido na coleta de dados possivelmente têm contribuição da exposição na altura do tórax e dos olhos (exposição durante a administração do radiofármaco). Tais resultados refletem a possibilidade de utilização do dosímetro de cristalino como forma de avaliação das rotinas dos IOEs, em termos de radioproteção.

Os valores estimados (potencial) de $\mathrm{H}_{\text {lens }}$ anual encontram-se na Figura 3.

Figura 3: Potencial de Dose Anual no Cristalino de cada IOE monitorado (identificado por numeração sequencial após a sigla da equipe a que pertence).

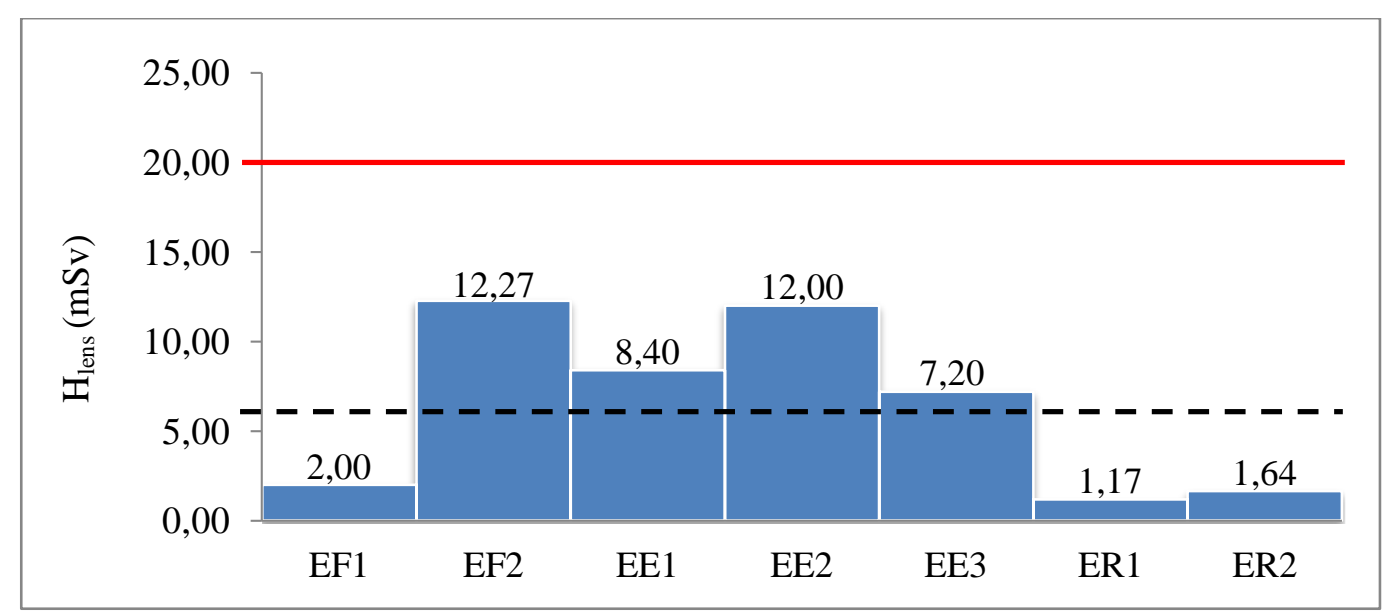


Com base nos resultados obtidos, seria improvável que o limite de $20 \mathrm{mSv}$ por ano (linha contínua) seja atingido pelos IOEs. Resultado condizente com a literatura $[6-8,10,12]$ de que o limite anual de dose no cristalino não seria excedido em decorrência unicamente da manipulação do F-18. Seria necessário que o EF2 e o EE2, profissionais com maiores $\mathrm{H}_{\text {lens, }}$, atendessem em torno de 160 pacientes/mês.

Todavia, quatro dos IOEs (toda a EE e o EF2) ultrapassam o nível de investigação de dose brasileiro para o cristalino (linha tracejada) de $6 \mathrm{mSv}$ por ano [14]. A IAEA orienta, no TECDOC n. 1731 [13], que deve-se implementar um programa rotineiro de monitoração de dose no cristalino caso o Potencial de Dose Anual ultrapasse $5 \mathrm{mSv}$. Logo, mesmo excluindo os dados do EF2 (profissional menos experiente), seria necessário, no SMN avaliado, a implementação de um programa de monitoração de dose no cristalino em decorrência da exposição dos IOEs na área de PET/CT.

Para a definição da necessidade (ou não) de utilização de monitor específico para cristalino, comparou-se a dose de cristalino à dose de corpo inteiro do IOE. Os resultados encontram-se na Tabela 2. A razão variou de 0,26-0,91 entre as equipes.

Tabela 2: Comparação entre os valores de dose efetiva de corpo inteiro e dose equivalente de cristalino recebidos por cada equipe de trabalho.

\begin{tabular}{cccc}
\hline Equipes & $\begin{array}{c}\mathbf{H}_{\text {lens,norm. }} \\
{[\boldsymbol{\mu S v} / \mathbf{G B q}(\boldsymbol{\mu S v} / \mathbf{m C i})]}\end{array}$ & $\begin{array}{c}\mathbf{E}_{\mathbf{n o r m} .} \\
{[\boldsymbol{\mu S v} / \mathbf{G B q}(\boldsymbol{\mu S v} / \mathbf{m C i})]}\end{array}$ & $\mathbf{R a z a ̃ o ~}_{\mathbf{l e n s} / \mathbf{E}}$ \\
\hline EF & $18,91(0,7)$ & $22,95(0,85)$ & 0,79 \\
$\mathbf{E E}$ & $23,89(0,88)$ & $27,34(1,01)$ & 0,91 \\
ER & $3,86(0,14)$ & $15,29(0,57)$ & 0,26 \\
\hline
\end{tabular}

Com base nesses valores, a $\mathrm{H}_{\text {lens }}$ dos IOEs das EF e EE poderia ser estimada a partir dos valores de $\mathrm{E}\left(\mathrm{H}_{\mathrm{lens}} \approx \mathrm{E}\right)$, não sendo necessária a utilização de dosímetro específico, uma vez que as razões obtidas aproximam-se de 1,0 [9]. Contudo, caso essa estimativa seja realizada para a ER, a $\mathrm{H}_{\text {lens }}$ será superestimada $\left(\mathrm{H}_{\mathrm{lens}}\right.$ corresponde a $26 \%$ da $\left.\mathrm{E}\right)$. 
Assim, considerando todas as equipes de trabalho da área de PET/CT do SMN do hospital avaliado, seria necessária a adoção de um dosímetro específico para a avaliação da dose recebida no cristalino dos IOEs da área. No entanto, levando-se em consideração o fator econômico ligado ao conceito de otimização da proteção radiológica [12], a adoção de um dosímetro específico (adicional aos habitualmente já utilizados em SMN) significaria um aumento de custo não justificável, uma vez que a $\mathrm{H}_{\text {lens }}$ da $\mathrm{ER}$ estaria sendo super, e não sub, estimada mediante a avaliação a partir dos valores de E.

\section{CONCLUSÕES}

Neste estudo avaliou-se a dose recebida no cristalino pelo IOE da área de PET/CT do SMN de um Hospital Escola público de porte especial. Os IOEs foram monitorados com dosímetros OSL, calibrados em $\mathrm{H}_{\mathrm{x}}$, para avaliação das doses equivalente de cristalino $\left(\mathrm{H}_{\text {lens }}\right)$ e efetiva de corpo inteiro (E), durante o período de um mês.

Baseado na estimativa da $\mathrm{H}_{\text {lens }}$ anual seria necessário a implementação de um programa rotineiro de monitoração de dose do cristalino, pois apesar de ser improvável que o limite de 20 mSv/ano seja atingido, 04 IOEs alcançariam o nível de investigação anual estabelecido pela CNEN [14], ultrapassando o limiar de Potencial de Dose Anual em que a IAEA recomenda a monitoração contínua [13].

A comparação entre os valores de $\mathrm{H}_{\text {lens }}$ e E indica que tal programa deveria prever o uso de um dosímetro específico para avaliação da $\mathrm{H}_{\text {lens. }}$. Todavia, a adoção de um dosímetro adicional significaria um aumento de custo não justificável levando-se em consideração o fator econômico relacionado ao conceito de otimização da radioproteção.

Assim, conclui-se que, nas condições de trabalho avaliadas, seria necessário que a $\mathrm{H}_{\text {lens }}$ dos IOEs seja constantemente avaliada (programa rotineiro de monitoração), mediante estimativa da $\mathrm{H}_{\text {lens }}$ a partir dos valores de $\mathrm{E}$ individuais.

Contudo, caso as condições de trabalho sejam alteradas, como, por exemplo, o SMN passe a utilizar, também, Ga-68 (radionuclídeo emissor de partículas beta positivas com energia média de $836 \mathrm{keV}$ e energia máxima de $1.899 \mathrm{MeV}$ ) para realização de PET/CT, torna-se necessária nova 
avaliação. A IAEA recomenda a utilização de dosímetros específicos, calibrados em $\mathrm{H}_{\mathrm{p}}(3)$, caso o IOE seja exposto à campos de radiação gama/beta mistos, cuja emissão beta máxima seja superior a $700 \mathrm{keV}[13]$.

Aponta-se como limitação deste estudo a utilização de monitores calibrados em Dose Individual, $\mathrm{H}_{\mathrm{x}}$. Recomenda-se que após os laboratórios de dosimetria brasileiros obterem a certificação do CASEC/IRD/CNEN para prestação de serviço de monitoração individual externa utilizando as grandezas operacionais $\mathrm{H}_{\mathrm{p}}(10)$ e $\mathrm{H}_{\mathrm{p}}(3)$ seja realizada nova avaliação.

\section{AGRADECIMENTO}

À empresa SAPRA LANDAUER - Serviços de Assessoria e Proteção Radiológica, particularmente à diretora-presidente Dr. Yvone Mascarenhas, pelo fornecimento dos dosímetros para realização desta pesquisa.

\section{REFERÊNCIAS}

[1] CNEN - Comissão Nacional de Energia Nuclear. Resolução $\mathbf{N}^{\circ}$ 114, de 24 de agosto de 2011. Brasil: D.O.U. 01/09/11. Disponível em:

$<$ http://pesquisa.in.gov.br/imprensa/jsp/visualiza/index.jsp?jornal=1\&pagina=31\&data=01/12/2 011>. Último acesso em: 14 Jun. 2018.

[2] ICRP - International Commission on Radiological Protection. Statement on Tissue Reactions. Ref. 4825-3093-1464, 2011. Disponível em: <http://www.icrp.org/page.asp?id=123>. Último acesso em: 14 Jun. 2018.

[3] CEMUSOVA, Z.; EKENDAHL, D.; JUDAS, L. Assessment of the Eye Lens Doses in Interventional Radiology: A Simulation in Laboratory Conditions. Radiation Protection Dosimetry, v. 170, 2016. Disponível em: <http://iopscience.iop.org/article/10.1088/09524746/33/2/445/meta>. Último acesso em: 14 Jun. 2018. 
[4] CNEN - Comissão Nacional de Energia Nuclear. Licenciamento e Controle de Instalações Medicinas. Brasil: 2017. Disponível em: <http://www.cnen.gov.br/orientacoes>. Último acesso em: 14 Jun. 2018.

[5] IRD - Instituto de Radioproteção e Dosimetria. Laboratórios Certificados para Prestação de Serviço de Monitoração Individual Externa. Rio de Janeiro: nov. 2017. Disponível em: <http://www.ird.gov.br/index.php/casec>. Último acesso em: 14 Jun. 2018.

[6] DABIN, J. et al. Eye Lens Doses in Nuclear Medicine: A Multicentric Study in Belgium and Poland. Radiation Protection Dosimetry, v. 170, 2016. Disponível em: <https://academic.oup.com/rpd/article/170/1-4/297/2223871>. Último acesso em: 14 Jun. 2018.

[7] MARINO, E. A. Optimización de la Dosis en Cristalino em Personal Técnico de um Servicio PET/CT. In: X CONGRESSO REGIONAL LATINOAMERICANO IRPA DE PROTEÇÃo E SEGURANÇA RADIOLÓGICA, 2015, Buenos Aires. Disponível em: <http://www.irpabuenosaires2015.org/Archivos/trcompletos/irpa/Marinofullpapertemplate_IRPA2015.pdf>. Último acesso em: 14 Jun. 2018.

[8] SZERMERSKI, B. et al. Dose rate constants for the quantity $\mathrm{H}_{\mathrm{p}}(3)$ for frequently used radionuclides in nuclear medicine. Z. Med. Phys, p. 7, nov 2015, ZEMEDI-10638. Disponível em: <https://www.sciencedirect.com/science/article/pii/S093938891500149X?via\%3Dihub>. Último acesso em: 14 Jun. 2018.

[9] KOPEC, R. et al. On the relationship between whole body, extremity and eye lens doses for medical staff in the preparation and application of radiopharmaceuticals in nuclear medicine. Radiation Measurements, v. 46, p. 1295-1298, 2011. Disponível em: <https://doi.org/10.1016/j.radmeas.2011.07.036>. Último acesso em: 14 Jun. 2018.

[10] KUBO, A.L.S.L.; MAURICIO, C.L.P. TLD Occupational dose distribution study in nuclear medicine. Radiation Measurements, ed. 71, p. 442-446, 2014. Disponível em: <https://doi.org/10.1016/j.radmeas.2014.04.021>. Último acesso em: 14 Jun. 2018.

[11] CNEN - Comissão Nacional de Energia Nuclear. Resolução No 102, de 22 de dezembro de 2010. Brasil: D.O.U. 10/05/11, p. 7-10, seção 1. Disponível em: 
$<$ http://pesquisa.in.gov.br/imprensa/jsp/visualiza/index.jsp?data=10/05/2011\&jornal=1\&pagina =7\&totalArquivos=192>. Último acesso em: 14 Jun. 2018.

[12] CNEN - Comissão Nacional de Energia Nuclear. Resolução No 229, de 03 de maio de 2018. Brasil: D.O.U. 07/05/18, p. 17, seção 1. Disponível em: $<$ http://www.imprensanacional.gov.br/materia//asset_publisher/Kujrw0TZC2Mb/content/id/13126946/do1-2018-05-07-resolucao-n-229-de-3de-maio-de-2018-13126942>. Último acesso em: 14 Jun. 2018.

[13] IAEA - International Atomic Energy Agency. Implications for Occupational Radiation Protection of the New Dose Limit for the Lens of the Eye. IAEA TECDOC 1731. IAEA, Viena, 2013. Disponível em: <https://www-pub.iaea.org/books/iaeabooks/10628/Implicationsfor-Occupational-Radiation-Protection-of-the-New-Dose-Limit-for-the-Lens-of-the-Eye> . Último acesso em: Último acesso em: 14 Jun. 2018.

[14] CNEN - Comissão Nacional de Energia Nuclear. Restrição de dose, níveis de referência ocupacionais e classificação de áreas. POSIÇÃO REGULATÓRIA 3.01/004:2011. Brasil: 2011. Disponível em: <http://www.cnen.gov.br/normas-tecnicas>. Último acesso em: 14 Jun. 2018. 